\title{
On approximating contractive systems
}

\author{
Meir Botner, Yoram Zarai, Michael Margaliot, and Lars Grüne
}

\begin{abstract}
We study the problem of approximating the trajectories of a contractive system using a simpler system (e.g. an LTI system). We derive bounds on the approximation error that depend on the trajectories of the simpler system. We describe several applications of our results to models from systems biology.
\end{abstract}

\section{Index Terms}

Contractive systems, matrix measures, linearized model, input to state dynamical stability, systems biology.

\section{INTRODUCTION}

A dynamical system is called contractive if any two trajectories contract to each other [1], [2]. This has many important implications. For example, if the trajectories evolve on a compact and convex state-space then the system admits an asymptotically globally stable equilibrium point $e$. Proving this does not require an explicit description of $e$. Also, contractive systems with a periodic excitation entrain, that is, their trajectories converge to a periodic pattern with the same period as the excitation (see, e.g. [3]).

Not surprisingly, contraction theory found numerous applications in nonlinear systems and control theory. A particularly interesting line of research is based on combining contraction

The research of MM is partially supported by research grants from the Israeli Ministry of Science, Technology \& Space and from the Israel Science Foundation.

M. Botner is with the School of Electrical Engineering, Tel-Aviv University, Tel-Aviv 69978, Israel. E-mail: meir.botner@gmail.com

Y. Zarai is with the School of Electrical Engineering, Tel-Aviv University, Tel-Aviv 69978, Israel. E-mail: yoramzaremail.tau.ac.il

M. Margaliot (Corresponding Author) is with the School of Electrical Engineering and the Sagol School of Neuroscience, Tel-Aviv University, Tel-Aviv 69978, Israel. E-mail: michaelmeeng.tau.ac.il

L. Grüne is with the Mathematical Institute, University of Bayreuth, 95440 Bayreuth, Germany. E-mail: lars.grueneduni-bayreuth. de 
theory and graph theory in order to study various networks of multi-agent systems (see, e.g. [4], $[5])$.

As already noted by Desoer and Haneda [6], contractive systems also satisfy an input-to-state stability (ISS) property. Desoer and Haneda used this to derive bounds on the error between trajectories of a continuous-time contractive system and its discretized model. This is important when computing solutions of contractive systems using numerical integration methods [7].

Here, we use the ISS perspective to derive a bound on the error between trajectories of a continuous-time contractive system and those of some "simpler" continuous-time system (e.g. an LTI system). This bound is particularly useful when the simpler model can be solved explicitly. We demonstrate our results using several models from systems biology.

The remainder of this note is organized as follows. The next section reviews some properties of contractive systems that are used later on. Section III describes our main results. The final section concludes and describes possible directions for further research.

\section{PRELIMINARIES}

Consider the time-varying dynamical system

$$
\dot{x}(t)=f(t, x(t))
$$

with the state $x$ evolving on a positively invariant convex set $\Omega \subseteq \mathbb{R}^{n}$. We assume that $f(t, x)$

is differentiable with respect to $x$, and that both $f(t, x)$ and $J(t, x):=\frac{\partial f}{\partial x}(t, x)$ are continuous in $(t, x)$. Let $x\left(t, t_{0}, x_{0}\right)$ denote the solution of (1) at time $t \geq t_{0}$ with the initial condition $x\left(t_{0}\right)=$ $x_{0}$. For the sake of simplicity, we assume from here on that $x\left(t, t_{0}, x_{0}\right)$ exists and is unique for all $t \geq t_{0} \geq 0$ and all $x_{0} \in \Omega$.

We say that (1) is contractive on $\Omega$ with respect to a norm $|\cdot|: \mathbb{R}^{n} \rightarrow \mathbb{R}_{+}$if there exists $\eta>0$ such that

$$
\left|x\left(t, t_{0}, a\right)-x\left(t, t_{0}, b\right)\right| \leq \exp \left(-\left(t-t_{0}\right) \eta\right)|a-b|
$$

for all $t \geq t_{0} \geq 0$ and all $a, b \in \Omega$. This means that any two trajectories contract to one another at an exponential rate $\eta$. This implies in particular that the initial condition is "quickly forgotten".

We focus here on exponential contraction with respect to a fixed vector norm because there exist easy to check sufficient conditions, based on matrix measures, guaranteeing that (2) holds. 
However, it should be noted that contraction can be defined in a more general way, for example with respect to a time- and space-varying norm [1] (see also [8]).

A vector norm $|\cdot|: \mathbb{R}^{n} \rightarrow \mathbb{R}_{+}$induces a matrix measure $\mu: \mathbb{R}^{n \times n} \rightarrow \mathbb{R}$ defined by

$$
\mu(A):=\lim _{\varepsilon \downarrow 0} \frac{1}{\varepsilon}(\|I+\varepsilon A\|-1),
$$

where $\|\cdot\|: \mathbb{R}^{n \times n} \rightarrow \mathbb{R}_{+}$is the matrix norm induced by $|\cdot|$. For example, for the $\ell_{1}$ vector norm, denoted $|\cdot|_{1}$, the induced matrix norm is the maximum absolute column sum of the matrix, and the induced matrix measure is

$$
\mu_{1}(A)=\max \left\{c_{1}(A), \ldots, c_{n}(A)\right\}
$$

where

$$
c_{j}(A):=A_{j j}+\sum_{\substack{1 \leq i \leq n \\ i \neq j}}\left|A_{i j}\right|,
$$

i.e., the sum of the entries in column $j$ of $A$, with non-diagonal elements replaced by their absolute values.

Matrix measures satisfy several useful properties (see, e.g. [6]). We list here two properties that will be used later on:

$$
\begin{aligned}
\mu(A+B) & \leq \mu(A)+\mu(B), & & \text { (subadditivity) } \\
\mu(c A) & =c \mu(A) \text { for all } c \geq 0, & & \text { (homogeneity) }
\end{aligned}
$$

Let $J(t, x):=\frac{\partial f}{\partial x}(t, x)$ denote the Jacobian of $f$. If

$$
\mu(J(t, x)) \leq-\eta, \quad \text { for all } x \in \Omega \text { and all } t \geq 0,
$$

then (2) holds (see [3] for a self-contained proof). This is in fact a particular case of using a Lyapunov-Finsler function to prove contraction [8]. We will focus on the case where $\eta>0$, but all our results hold when $\eta \leq 0$ as well.

Often it is useful to work with scaled norms (see, e.g. [9]). Let $|\cdot|_{*}$ be some vector norm, and let $\mu_{*}: \mathbb{R}^{n \times n} \rightarrow \mathbb{R}$ denote its induced matrix measure. If $D \in \mathbb{R}^{n \times n}$ is an invertible matrix, and $|\cdot|_{*, D}: \mathbb{R}^{n} \rightarrow \mathbb{R}_{+}$is the vector norm defined by $|z|_{*, D}:=|D z|$ then the induced matrix measure is $\mu_{*, D}(A)=\mu\left(D A D^{-1}\right)$. 


\section{MAIN Results}

From here on we assume that the Jacobian $J$ of the vector field $f$ in (1) satisfies

$$
\mu(J(t, x)) \leq-\eta, \text { for all } x \in \Omega \text { and all } t \geq 0,
$$

where $\mu$ is the matrix measure induced by a norm $|\cdot|$. We can now state our main result. This provides bounds on the error between trajectories of a contractive system and those of some other ("simpler") dynamical system.

Theorem 1 Consider system (1), with $x(0)=x_{0} \in \Omega$, and suppose that (7) holds for a matrix measure induced by some norm $|\cdot|$. Consider another system $\dot{y}(t)=g(t, y)$, with $y(0)=y_{0} \in \Omega$. Let $T=T\left(y_{0}\right) \geq 0$ be such that $y\left(t, 0, y_{0}\right) \in \Omega$ for all $t \in[0, T]$. Let $d\left(t, x_{0}, y_{0}\right):=x\left(t, 0, x_{0}\right)-$ $y\left(t, 0, y_{0}\right)$ denote the difference between the trajectories at time $t$. Then for any $t \in[0, T]$ the following inequalities hold:

$$
\begin{aligned}
& \left|d\left(t, x_{0}, y_{0}\right)\right| \leq \exp (-\eta t)\left|x_{0}-y_{0}\right| \\
& +\int_{0}^{t} \exp (-\eta(t-s))\left|f\left(s, y\left(s, 0, y_{0}\right)\right)-g\left(s, y\left(s, 0, y_{0}\right)\right)\right| \mathrm{d} s
\end{aligned}
$$

and

$$
\begin{aligned}
& \left|d\left(t, x_{0}, y_{0}\right)\right| \leq \exp (-\eta(1-\alpha) t) \times \max \left\{\left|x_{0}-y_{0}\right|,\right. \\
& \left.\sup _{\tau \in[0, t]} \frac{\exp (\eta(1-\alpha) \tau)}{\eta \alpha}\left|f\left(\tau, y\left(\tau, 0, y_{0}\right)\right)-g\left(\tau, y\left(\tau, 0, y_{0}\right)\right)\right|\right\}
\end{aligned}
$$

for all $\alpha \in(0,1)$.

The advantage of the bounds (8) and (9) is that they depend on the difference in the vector fields $f-g$ evaluated along the solution of the $y$-system. As we will see below, this is particularly useful when either the solution $y(t)$ is "simple", e.g., when the $y$ dynamics is LTI, or when the difference $|f-g|$ admits a simple bound. In fact, the bounds demonstrate a tradeoff: if $g$ is "close" to $f$ then the difference $|f(y)-g(y)|$ will be small yet the solution $y$ of the $g$ system will be difficult to evaluate explicitly (as we assume that $f$ is a nonlinear vector field). On the other-hand, if the vector field $g$ is "simple" (e.g. LTI), then the distance $|f(y)-g(y)|$ may be large yet the $y$ solution may be known explicitly. 
While the estimate (9) may be more conservative than the integral estimate in (8), it can be useful when the integral does not allow for an explicit solution.

Proof of Theorem 1. Note that

$$
\begin{aligned}
\dot{x}(t)-\dot{y}(t) & =f(t, x(t))-f(t, y(t))+f(t, y(t))-g(t, y(t)) \\
& =M(t)(x(t)-y(t))+u(t)
\end{aligned}
$$

where $M(t):=\int_{0}^{1} J(t, s x(t)+(1-s) y(t)) \mathrm{d} s$, and $u(t):=f(t, y(t))-g(t, y(t))$. Since $y(t) \in \Omega$ for all $t \in[0, T]$ and $\Omega$ is convex, $s x(t)+(1-s) y(t) \in \Omega$ for all $t \in[0, T]$ and all $s \in[0,1]$. Now (5) and (6) imply that $\mu(M(t)) \leq-\eta$ for all $t \in[0, T]$. Using the ISS property of contractive systems [6] yields

$$
\begin{aligned}
\left|d\left(t, x_{0}, y_{0}\right)\right| \leq & \exp (-\eta t)\left|x_{0}-y_{0}\right| \\
& +\int_{0}^{t} \exp (-\eta(t-s))|u(s)| \mathrm{d} s,
\end{aligned}
$$

and this proves (8).

To prove (9), we use the concept of input-to-state dynamical stability (ISDS) as introduced in [10] and [11]. We first briefly review some definitions. Consider a function $V: P \rightarrow \mathbb{R}$, with $P \subseteq \mathbb{R}^{n}$. Recall that the viscosity subdifferential of $V$ at a point $z \in P$, denoted $D^{-} V(z)$, is the set of all vectors $p \in \mathbb{R}^{n}$ satisfying

$$
\liminf _{\hat{z} \rightarrow z} \frac{V(\hat{z})-V(z)-p(\hat{z}-z)}{|\hat{z}-z|} \geq 0
$$

The function $V$ is called a viscosity supersolution of the equation $H(x, V, D V)=0, x \in P$, if $V$ is upper semicontinuous and

$$
H(x, V(x), p) \geq 0, \quad \text { for all } x \in P, p \in D^{-} V(x) .
$$

We will prove that $V(z):=|z|$ is an ISDS Lyapunov function in the sense of Definition 3.5.2 in [11] for the system $\dot{z}(t)=M(t) z(t)+w(t)$, with the functions: $\sigma(r):=r, \gamma(r):=r /(\eta \alpha)$, and $\mu(r, t):=\exp (-\eta(1-\alpha) t) r$. Note that $\frac{d}{d t} \mu(r, t)=-g(\mu(r, t))$, with $g(s):=\eta(1-\alpha) s$. Then [11, Proposition 3.5.1] yields (9). Specifically, we need to establish that $V$ is a viscosity 
supersolution of the equation:

$$
\inf _{w \in \mathbb{R}^{n}, \gamma(|w|) \leq V(z)}\{-D V(z)(M(t) z+w)-g(V(z))\}=0 .
$$

This is equivalent to the following property: for each $z \in \mathbb{R}^{n}, t \geq 0$ and $p \in D^{-} V(z)$,

$$
\sup _{w \in \mathbb{R}^{n}, \gamma(|w|) \leq V(z)} p(M(t)+w) z \leq-g(V(z)) .
$$

To show this, note that for $p \in D^{-} V(z)$ we have that for each $w \in \mathbb{R}^{n}$ satisfying $M(t) z+w \neq 0$,

$$
\liminf _{h \rightarrow 0} \frac{V(z+h M(t) z+h w)-V(z)-h p(M(t) z+w)}{|h(M(z) z+w)|} \geq 0,
$$

that is

$$
\begin{aligned}
p(M(t) z+w) & \leq \liminf _{h \rightarrow 0} \frac{V(z+h M(t) z+h w)-V(z)}{h} \\
& =\liminf _{h \rightarrow 0} \frac{|z+h M(t) z+h w|-|z|}{h} \\
& \leq \liminf _{h \rightarrow 0} \frac{\| I+h M(t)|||z|+h|w|-|z|}{h} \\
& \leq \mu(M(t))|z|+|w| \\
& \leq-\eta V(z)+|w|,
\end{aligned}
$$

where we used the definition of the matrix measure and (7).

Now for any $w$ such that $\gamma(|w|) \leq V(z)$ we obtain $|w| \leq \alpha \eta V(z)$ which implies

$$
\begin{aligned}
-\eta V(z)+|w| & \leq-\eta V(z)+\alpha \eta V(z) \\
& =-\eta(1-\alpha) V(z) \\
& =-g(V(z)),
\end{aligned}
$$

and combining this with (12) yields (11).

The next two sections describe several applications of Theorem 1.

\section{A. Contractive systems with perturbations}

One case where Theorem 1 is useful is when the difference $|f-g|$ can be efficiently bounded. Sontag [12] has shown that if $\dot{x}=f(t, x)$ is contractive and $h(t)$ is an exponentially decaying 
function then there exist $k, \ell$, with $\ell>0$, such that the difference between the solutions of

$$
\dot{x}=f(t, x), \quad x(0)=x_{0},
$$

and

$$
\dot{y}=f(t, y)+h(t), \quad y(0)=y_{0},
$$

satisfies

$$
\left|x\left(t, x_{0}\right)-y\left(t, y_{0}\right)\right| \leq \exp (-\ell t)\left(k+\left|x_{0}-y_{0}\right|\right) .
$$

This is a kind of a "exponentially converging input exponentially converging output" property for contractive systems. We provide a more explicit bound.

Corollary 1 Suppose that trajectories of (13) evolve on a convex set $\Omega \subseteq \mathbb{R}^{n}$ and that its Jacobian satisfies $\mu(J(t, x))<-\eta$ for all $x \in \Omega$ and all $t \geq 0$. Here $\mu$ is a matrix measure induced by a vector norm $|\cdot|$. Suppose also that $h(t)$ in (14) satisfies $|h(t)| \leq L \exp (-k t)$ for all $t \geq 0$ where $k>0$ and $L \geq 0$. Let $T \geq 0$ be such that $y\left(t, y_{0}\right) \in \Omega$ for all $t \in[0, T]$. Then the difference between the solutions of (13) and (14) satisfies for all $t \in[0, T]$,

$$
\left|d\left(t, x_{0}, y_{0}\right)\right| \leq \exp (-\eta t)\left|x_{0}-y_{0}\right|+L b(t),
$$

where

$$
b(t):= \begin{cases}t \exp (-\eta t), & \eta=k, \\ \frac{\exp (-k t)-\exp (-\eta t)}{\eta-k}, & \eta \neq k .\end{cases}
$$

Indeed, Theorem 1 yields

$$
\begin{aligned}
& \left|d\left(t, x_{0}, y_{0}\right)\right| \\
& \leq \exp (-\eta t)\left|x_{0}-y_{0}\right|+\int_{0}^{t} \exp (-\eta(t-s))|h(s)| \mathrm{d} s \\
& \leq \exp (-\eta t)\left|x_{0}-y_{0}\right|+L \int_{0}^{t} \exp (-\eta(t-s)) \exp (-k s) \mathrm{d} s,
\end{aligned}
$$

and this yields (15).

Let $1_{k}$ denote the vector of $k$ ones. As a simple example of (15), consider the case where $f(t, x)=$ $-x$ and $h(t)=\exp (-t) 1_{n}$. Here, $\mu_{1}(J(t, x))=-1$ for all $t \geq 0$ and all $x \in \mathbb{R}^{n}$, and $|h(t)|_{1}=$ 
$n \exp (-t)$. Applying Corollary 1 with $\eta=k=1, L=n$, and initial conditions $x_{0}=y_{0}=0$ yields

$$
|x(t, 0)-y(t, 0)|_{1} \leq n t \exp (-t)
$$

and this is in fact a tight bound, as in this case $x(t, 0) \equiv 0$ and $y(t, 0)=t \exp (-t) 1_{n}$.

Theorem 1 can also be used to obtain bounds for the case where $h$ decays at a rate that is slower than exponential (e.g. when $|h(t)| \leq L \frac{1}{1+t}$ ). However, a more interesting application of Theorem 1 is when the approximating system (the $y$ system) is an LTI system.

\section{B. Approximation using an LTI system}

As a first application, consider the cyclic system

$$
\begin{aligned}
& \dot{x}_{1}=-\alpha_{1} x_{1}+h\left(x_{n}\right), \\
& \dot{x}_{2}=-\alpha_{2} x_{2}+x_{1}, \\
& \dot{x}_{3}=-\alpha_{3} x_{3}+x_{2}, \\
& \quad \vdots \\
& \dot{x}_{n}=-\alpha_{n} x_{n}+x_{n-1},
\end{aligned}
$$

with $\alpha_{i}>0$. We assume that the "feedback function" $h$ satisfies: $h(0) \geq 0$, and there exists $c>0$ such that

$$
0 \leq h^{\prime}(z) \leq\left(\alpha_{1}-c\right) \ldots\left(\alpha_{n}-c\right), \quad \text { for all } z \geq 0
$$

As explained in [13, Ch. 4], a system in the form (16) may model a simple biochemical feedback control circuit for protein synthesis in the cell. The $x_{i}$ 's represent concentrations of various macro-molecules in the cell and are therefore non-negative. It is straightforward to verify that $\mathbb{R}_{+}^{n}$ is an invariant set of (16), and we assume that there exists a compact and convex subset $\Omega \subset \mathbb{R}_{+}^{n}$ that is also an invariant set of the dynamics. 
The Jacobian of (16) is

$$
J(x)=\left[\begin{array}{cccccc}
-\alpha_{1} & 0 & 0 & \ldots & 0 & h^{\prime}\left(x_{n}\right) \\
1 & -\alpha_{2} & 0 & \ldots & 0 & 0 \\
0 & 1 & -\alpha_{3} & \ldots & 0 & 0 \\
& & \vdots & & & \\
0 & 0 & 0 & \ldots & 1 & -\alpha_{n}
\end{array}\right] .
$$

For the diagonal matrix $D=D(\varepsilon):=\operatorname{diag}\left(1, \alpha_{1}-\varepsilon,\left(\alpha_{1}-\varepsilon\right)\left(\alpha_{2}-\varepsilon\right), \ldots,\left(\alpha_{1}-\varepsilon\right) \ldots\left(\alpha_{n-1}-\varepsilon\right)\right)$, with $\varepsilon \in\left[0, \min \left\{\alpha_{i}\right\}\right)$, the matrix $D J(x) D^{-1}$ has the form

$$
\left[\begin{array}{cccccc}
-\alpha_{1} & 0 & 0 & \ldots & 0 & \frac{h^{\prime}\left(x_{n}\right)}{\prod_{i=1}^{n-1}\left(\alpha_{i}-\varepsilon\right)} \\
\alpha_{1}-\varepsilon & -\alpha_{2} & 0 & \ldots & 0 & 0 \\
0 & \alpha_{2}-\varepsilon & -\alpha_{3} & \ldots & 0 & 0 \\
& \vdots & & & \\
0 & 0 & 0 & \ldots & \alpha_{n-1}-\varepsilon & -\alpha_{n}
\end{array}\right],
$$

and our assumptions on $h(\cdot)$ imply that for any $\varepsilon \in[0, c], \mu_{1}\left(D J(x) D^{-1}\right)=-\varepsilon$, for all $x \in \mathbb{R}_{+}^{n}$. Thus, (16) is contractive on $\Omega$ w.r.t. the scaled norm $|\cdot|_{1, D_{\varepsilon}}$, with contraction rate $\varepsilon$. Intuitively speaking, condition (17) thus requires that the "total dissipation" $\alpha:=\prod_{i=1}^{n} \alpha_{i}$ is strictly larger than the maximal value of the derivative of the feedback function.

A linear system that can be used to approximate (16) is

$$
\begin{aligned}
& \dot{y}_{1}=-\alpha_{1} y_{1}, \\
& \dot{y}_{2}=-\alpha_{2} y_{2}+y_{1}, \\
& \dot{y}_{3}=-\alpha_{3} y_{3}+y_{2}, \\
& \vdots \\
& \dot{y}_{n}=-\alpha_{n} y_{n}+y_{n-1} .
\end{aligned}
$$

Indeed, $\mathbb{R}_{+}^{n}$ is an invariant set of this system, and moreover it admits a closed-form solution. To 
simplify the presentation, assume that all the $\alpha_{i}$ s are equal. Then this closed-form solution is

$$
y(t)=\exp \left(-\alpha_{1} t\right)\left[\begin{array}{ccccc}
1 & 0 & 0 & \ldots & 0 \\
t & 1 & 0 & \ldots & 0 \\
\frac{t^{2}}{2} & t & 1 & \ldots & 0 \\
& & \vdots & & \\
\frac{t^{n-1}}{(n-1) !} & \frac{t^{n-2}}{(n-2) !} & \frac{t^{n-3}}{(n-3) !} & \ldots & 1
\end{array}\right] y(0)
$$

Pick an initial condition $x(0)=y(0) \in \mathbb{R}_{+}^{n}$. Assume that $y\left(t, x_{0}\right) \in \Omega$ for all $t \in[0, T]$. Then the bound (8) implies that for any $\varepsilon \in[0, c]$ and any $t \in[0, T]$,

$$
\begin{aligned}
& |x(t, x(0))-y(t, x(0))|_{1, D_{\varepsilon}} \\
& \leq \int_{0}^{t} \exp (-\varepsilon(t-s))\left|\left[\begin{array}{llll}
h\left(y_{n}(s)\right) & 0 & \ldots & 0
\end{array}\right]^{\prime}\right|_{1, D_{\varepsilon}} \mathrm{d} s \\
& =\int_{0}^{t} \exp (-\varepsilon(t-s))\left|D_{\varepsilon}\left[\begin{array}{llll}
h\left(y_{n}(s)\right) & 0 & \ldots & 0
\end{array}\right]^{\prime}\right|_{1} \mathrm{~d} s \\
& =\int_{0}^{t} \exp (-\varepsilon(t-s)) h\left(y_{n}(s)\right) \mathrm{d} s \\
& =\int_{0}^{t} \exp (-\varepsilon(t-s)) h\left(\exp \left(-\alpha_{1} s\right) \sum_{k=1}^{n} \frac{s^{n-k}}{(n-k) !} y_{k}(0)\right) \mathrm{d} s .
\end{aligned}
$$

Thus, we obtain an explicit integrand in the integral bound for the error between the trajectories of the original nonlinear contractive system and the simpler linear system.

As a specific application, consider the case where the initial condition is $x(0)=y(0)=0$. Then the solution of (19) is $y(t) \equiv 0$ for all $t \geq 0$, so (21) yields

$$
\begin{aligned}
\left|D_{\varepsilon} x(t, 0)\right|_{1} & \leq h(0) \int_{0}^{t} \exp (-\varepsilon(t-s)) \mathrm{d} s \\
& =h(0) \frac{1-\exp (-\varepsilon t)}{\varepsilon} .
\end{aligned}
$$

This gives a simple bound on how far can the solution of (16) escape from the origin, and thus also a bound on the location of the equilibrium point of (16) in $\mathbb{R}_{+}^{n}$.

The next result specializes Theorem 1 for the case where $f$ is time-invariant, and the $y$ system is obtained by linearizing it around some point $z \in \Omega$. We assume, w.1.o.g., that $z=0$. 
Corollary 2 Consider the system

$$
\dot{x}=f(x),
$$

and suppose that its Jacobian satisfies (6) where $\mu$ is a matrix measure induced by a norm $|\cdot|$. Let $x(t)$ denote the solution of this system at time $t$ for $x(0)=0$. Let $f(x)=b+A x+v(x)$, where $v(x)$ contains only non-linear terms in $x$. Consider the linearized system $\dot{y}=A y+b$, $y(0)=0$. Let $T \geq 0$ be such that $y(t) \in \Omega$ for all $t \in[0, T]$. Then for all $t \in[0, T]$,

$$
|x(t)-y(t)| \leq \int_{0}^{t} \exp (-\eta(t-s))|v(y(s))| \mathrm{d} s .
$$

Indeed, Thm. 1 implies that

$$
|x(t)-y(t)| \leq \int_{0}^{t} \exp (-\eta(t-s))|f(y(s))-A y(s)-b| \mathrm{d} s,
$$

and this yields (23).

Note that if $A$ is invertible then $y(s)$ in (23) satisfies $y(s)=A^{-1}(\exp (A s)-I) b$.

Corollary 2 provides a bound on the error between the trajectories of a contractive system and its linearization. Since $y(t)$ is the solution of an LTI system, there are cases where this leads to an explicit bound. The next example demonstrates this.

Example 1 The ribosome flow model (RFM) [14] is a nonlinear compartmental model describing the unidirectional flow of particles along a chain of $n$ sites using $n$ non-linear first-order differential equations:

$$
\begin{aligned}
\dot{x}_{1} & =\lambda_{0}\left(1-x_{1}\right)-\lambda_{1} x_{1}\left(1-x_{2}\right), \\
\dot{x}_{2} & =\lambda_{1} x_{1}\left(1-x_{2}\right)-\lambda_{2} x_{2}\left(1-x_{3}\right), \\
\dot{x}_{3} & =\lambda_{2} x_{2}\left(1-x_{3}\right)-\lambda_{3} x_{3}\left(1-x_{4}\right), \\
& \vdots \\
\dot{x}_{n-1} & =\lambda_{n-2} x_{n-2}\left(1-x_{n-1}\right)-\lambda_{n-1} x_{n-1}\left(1-x_{n}\right), \\
\dot{x}_{n} & =\lambda_{n-1} x_{n-1}\left(1-x_{n}\right)-\lambda_{n} x_{n} .
\end{aligned}
$$

Here $x_{i}(t) \in[0,1]$ represents the level of occupancy of site $i$ at time $t$, normalized such that $x_{i}(t)=1\left[x_{i}(t)=0\right]$ means that site $i$ is completely full [empty]. It is straightforward 
to verify that $[0,1]^{n}$ is an invariant set of (24). The transition rate $\lambda_{i}>0$ controls the flow from site $i$ to site $i+1$, with $\lambda_{0}\left[\lambda_{n}\right]$ called the initiation [exit] rate. Note that the flow satisfies a "soft" excluded volume principle, that is, as site $i$ becomes fuller the flow from site $i-1$ to site $i$ decreases. This models the fact that the particles have volume and thus cannot overtake one another. The rate at which particles leave the chain, that is, $R(t):=\lambda_{n} x_{n}$ is called the production rate.

Recently, the RFM as been used to model and analyze the flow of ribosomes (the particles) along groups of codons (the sites) along the mRNA molecule during translation (see, e.g. [15], [16], [17], [18], [19], [20], [21]). In this case, every ribosome that leaves the chain releases the produced protein, so $R(t)$ is the protein production rate at time $t$.

In eukaryotic cells, the mRNA is produced in the cell nucleus and then transferred to the cytoplasm [22]. Only there can the first ribosomes attach to the mRNA. To study translation in such a newly created mRNA molecule, it is natural to consider the RFM with $x(0)=0$, i.e. when initially there are no ribosomes on the mRNA molecule.

The linearization of the RFM around zero is:

$$
\dot{y}=A y+b,
$$

with

$$
A:=\left[\begin{array}{cccccc}
-\lambda_{0}-\lambda_{1} & 0 & 0 & 0 & \ldots & 0 \\
\lambda_{1} & -\lambda_{2} & 0 & 0 & \ldots & 0 \\
0 & \lambda_{2} & -\lambda_{3} & 0 & \ldots & 0 \\
& & & \vdots & & \\
0 & 0 & 0 & \ldots & \lambda_{n-1} & -\lambda_{n}
\end{array}\right]
$$

and $b:=\left[\begin{array}{llll}\lambda_{0} & 0 & \ldots & 0\end{array}\right]^{\prime}$. Note that just like the RFM, the system (25) is a tridiagonal cooperative dynamical system [23], so if $p, q \in \mathbb{R}^{n}$, with $p \leq q$ (i.e. $p_{i} \leq q_{i}$, for all $i=1, \ldots, n$ ), then $y(t, p) \leq y(t, q)$ for all $t \geq 0$. However, $[0,1]^{n}$ is not necessarily an invariant set of the $y$ dynamics. 
The nonlinear remainder is

$$
\begin{aligned}
v(x)= & {\left[\begin{array}{lll}
\lambda_{1} x_{1} x_{2} & -\lambda_{1} x_{1} x_{2}+\lambda_{2} x_{2} x_{3} & \ldots \\
& -\lambda_{n-2} x_{n-2} x_{n-1}+\lambda_{n-1} x_{n-1} x_{n}-\lambda_{n-1} x_{n-1} x_{n}
\end{array}\right]^{\prime} . }
\end{aligned}
$$

The eigenvalues of $A$ are: $\alpha_{1}:=-\lambda_{0}-\lambda_{1}, \alpha_{2}:=-\lambda_{2}, \ldots, \alpha_{n}:=-\lambda_{n}$. When all the $\alpha_{i}$ s are distinct the solution of (25), for $y(0)=0$, is

$$
\begin{aligned}
& y_{1}(t)=\frac{\lambda_{0}}{\alpha_{1}}\left(\exp \left(\alpha_{1} t\right)-1\right) \\
& y_{j}(t)=\left(\prod_{\ell=0}^{j-1} \lambda_{\ell}\right) \sum_{i=1}^{j} \frac{\exp \left(\alpha_{i} t\right)-1}{\alpha_{i} \prod_{\substack{k=1 \\
k \neq i}}^{j}\left(\alpha_{i}-\alpha_{k}\right)}, j=2, \ldots, n .
\end{aligned}
$$

The RFM is contractive w.r.t. the $\ell_{1}$ vector norm with rate $\eta=0 .{ }^{1}$ All this explicit information can now be plugged in the bound (23). To demonstrate this, we consider the case $n=2$. In this case, the Jacobian of (24) is

$$
J(x)=\left[\begin{array}{cc}
-\lambda_{0}-\lambda_{1}\left(1-x_{2}\right) & \lambda_{1} x_{1} \\
\lambda_{1}\left(1-x_{2}\right) & -\lambda_{1} x_{1}-\lambda_{2}
\end{array}\right] .
$$

For any $x \in[0,1]^{2}$ the off-diagonal terms here are non-negative, so $\mu_{1}(J(x))=\max \left\{-\lambda_{0},-\lambda_{2}\right\}$ for all $x \in[0,1]^{2}$. Thus, the RFM with $n=2$ is contractive w.r.t. the $\ell_{1}$ norm with contraction rate $\eta:=\min \left\{\lambda_{0}, \lambda_{2}\right\}>0$. The linearized model with $y(0)=0$ admits the solution

$$
\begin{aligned}
y_{1}(t) & =\frac{\lambda_{0}}{\lambda_{0}+\lambda_{1}}\left(1-\exp \left(-\left(\lambda_{0}+\lambda_{1}\right) t\right)\right), \\
y_{2}(t) & =\frac{\lambda_{0} \lambda_{1}}{\lambda_{2}-\lambda_{0}-\lambda_{1}} \\
& \times\left(\frac{1-\exp \left(-\left(\lambda_{0}+\lambda_{1}\right) t\right)}{\lambda_{0}+\lambda_{1}}-\frac{1-\exp \left(-\lambda_{2} t\right)}{\lambda_{2}}\right),
\end{aligned}
$$

where we assume that $\lambda_{0}+\lambda_{1} \neq \lambda_{2}$. Note that this implies that if $\lambda_{0} \lambda_{1} \leq \lambda_{2}\left(\lambda_{0}+\lambda_{1}\right)$ then $[0,1]^{2}$

${ }^{1}$ The RFM is also an "almost" contractive system in the sense defined in [24]. 
is an invariant set of the $y$ system. The bound (23) becomes

$$
\begin{aligned}
& |x(t, 0)-y(t, 0)|_{1} \\
& \leq \int_{0}^{t} e^{-\eta(t-s)}\left|\lambda_{1}\left[y_{1}(s) y_{2}(s)-y_{1}(s) y_{2}(s)\right]^{\prime}\right|_{1} \mathrm{~d} s \\
& =2 \lambda_{1} \int_{0}^{t} e^{-\eta(t-s)} y_{1}(s) y_{2}(s) \mathrm{d} s,
\end{aligned}
$$

for all $t \in[0, T]$. Plugging in (26) and integrating leads to a closed-form expression (that is omitted due to space limitations).

Note that (26) yields

$$
\begin{aligned}
& y_{1}(t)=\lambda_{0} t-\frac{\lambda_{0}\left(\lambda_{0}+\lambda_{1}\right)}{2} t^{2}+\frac{\lambda_{0}\left(\lambda_{0}+\lambda_{1}\right)^{2}}{6} t^{3}+O\left(t^{4}\right), \\
& y_{2}(t)=\frac{\lambda_{0} \lambda_{1}}{2} t^{2}-\frac{\lambda_{0} \lambda_{1}\left(\lambda_{0}+\lambda_{1}+\lambda_{2}\right)}{6} t^{3}+O\left(t^{4}\right),
\end{aligned}
$$

and so the bound (27) yields $|x(t, 0)-y(t, 0)|_{1}=O\left(t^{4}\right)$, implying that for small values of $t$ the explicit solution (26) of the linear model provides a good approximation of the unknown solution of the nonlinear RFM model with $n=2$. In particular, the production rate $R(t)=\lambda_{2} x_{2}(t)$ can be approximated by $\lambda_{2} y_{2}(t)=\frac{\lambda_{0} \lambda_{1} \lambda_{2}}{2} t^{2}-\frac{\lambda_{0} \lambda_{1} \lambda_{2}\left(\lambda_{0}+\lambda_{1}+\lambda_{2}\right)}{6} t^{3}+O\left(t^{4}\right)$. This shows how the various parameters affect the (short time) production rate.

Remark 1 When $\dot{y}=A y+b$ it is possible to obtain a different bound on the linearization error by noting that

$$
\begin{aligned}
\dot{x}-\dot{y} & =f(x)-A y-b \\
& =A(x-y)+f(x)-A x-b .
\end{aligned}
$$

Pick a vector norm $|\cdot|_{*}$ and let $\mu_{*}$ denote the induced matrix measure. Pick $\alpha \in \mathbb{R}$ such that

$$
\mu_{*}(A) \leq \alpha
$$


Then arguing as in the proof of Theorem 1 yields

$$
\begin{aligned}
|x(t)-y(t)|_{*} & \leq \int_{0}^{t} \exp (\alpha(t-s))|f(x(s))-A x(s)-b|_{*} \mathrm{~d} s \\
& =\int_{0}^{t} \exp (\alpha(t-s))|v(x(s))|_{*} \mathrm{~d} s .
\end{aligned}
$$

If $A$ is Hurwitz then we can always find a vector norm for which (29) holds with an $\alpha<0$. However, the bound (30) is less explicit than the bounds derived above, as it depends on the solution $x(s)$ of the nonlinear system (22) that is usually unknown.

Another natural idea is to use our results in order to analyze the behavior of (22) in the vicinity of an equilibrium point $e$. Without loss of generality, we may assume that $e=0$. Specializing Corollary 2 to this case yields the following result.

Corollary 3 Consider the system (22) and suppose that (6) holds for a matrix measure induced by some norm $|\cdot|$. Suppose that 0 is an equilibrium point of (22), and let $f(x)=A x+v(x)$, where $v(x)$ contains only non-linear terms in $x$. Pick $\varepsilon \in \Omega$. Consider the linearized system $\dot{y}=$ $A y, y(0)=\varepsilon$. Let $T \geq 0$ be such that $y(t, \varepsilon) \in \Omega$ for all $t \in[0, T]$. Then for all $t \in[0, T]$,

$$
|x(t, \varepsilon)-y(t, \varepsilon)| \leq \int_{0}^{t} e^{-\eta(t-s)}|v(\exp (A s) \varepsilon)| \mathrm{d} s .
$$

The next example demonstrates this bound.

Example 2 A basic model for a transcriptional module that is ubiquitous in both biology and synthetic biology is given by (see, e.g., [25], [3]):

$$
\begin{aligned}
& \dot{x}_{1}=-\delta x_{1}+k_{1} x_{2}-k_{2}\left(e_{T}-x_{2}\right) x_{1}, \\
& \dot{x}_{2}=-k_{1} x_{2}+k_{2}\left(e_{T}-x_{2}\right) x_{1},
\end{aligned}
$$

where $\delta, k_{1}, k_{2}, e_{T}>0$. Here $x_{1}(t)$ is the concentration at time $t$ of a transcriptional factor $X$ that regulates a downstream transcriptional module by binding to a promoter with concentration $e(t)$ yielding a protein-promoter complex $Y$ with concentration $x_{2}(t)$. The binding reaction is reversible with binding and dissociation rates $k_{2}$ and $k_{1}$, respectively. The linear degradation rate of $X$ is $\delta$, and as the promoter is not subject to decay, its total concentration, $e_{T}$, is conserved, so $e(t)=e_{T}-x_{2}(t)$ for all $t \geq 0$. 
Trajectories of (32) evolve on $[0, \infty) \times\left[0, e_{T}\right]$, and $\Omega:=\left[0, k_{1} e_{T} / \delta\right] \times\left[0, e_{T}\right]$ is a convex and compact invariant set.

Ref. [3] has shown that (32) is contractive w.r.t. a certain weighted $L_{1}$ norm. Indeed, the Jacobian of (32) is

$$
J(x)=\left[\begin{array}{cc}
-\delta-k_{2}\left(e_{T}-x_{2}\right) & k_{1}+k_{2} x_{1} \\
k_{2}\left(e_{T}-x_{2}\right) & -k_{1}-k_{2} x_{1}
\end{array}\right],
$$

and for $D=\operatorname{diag}(d, 1)$, with $d>0$,

$$
D J(x) D^{-1}=\left[\begin{array}{cc}
-\delta-k_{2}\left(e_{T}-x_{2}\right) & \left(k_{1}+k_{2} x_{1}\right) d \\
k_{2}\left(e_{T}-x_{2}\right) / d & -k_{1}-k_{2} x_{1}
\end{array}\right] .
$$

The off-diagonal terms here are non-negative, so $\mu_{1}\left(D J(x) D^{-1}\right)$ is equal to

$$
\max \left\{-\delta-k_{2}\left(e_{T}-x_{2}\right)\left(1-d^{-1}\right),-(1-d)\left(k_{1}+k_{2} x_{1}\right)\right\}
$$

This means that for any $d \in\left(\frac{k_{2} e_{T}}{k_{2} e_{T}+\delta}, 1\right)$,

$$
\mu_{1, D}(J(x)) \leq-\eta, \text { for all }\left[\begin{array}{ll}
x_{1} & x_{2}
\end{array}\right]^{\prime} \in \Omega
$$

where $\eta:=\min \left\{k_{1}(1-d), \delta+k_{2} e_{T}\left(1-d^{-1}\right)\right\}>0$. Thus, (32) is contractive with respect to the scaled norm $|\cdot|_{1, D}$ with contraction rate $\eta$.

Clearly, the origin is an equilibrium point of (32) (and the contractivity implies that this equilibrium is globally asymptotically stable). The linearized model is $\dot{y}=A y$, with

$$
A=\left[\begin{array}{cc}
-\delta-k_{2} e_{T} & k_{1} \\
k_{2} e_{T} & -k_{1}
\end{array}\right]
$$

and the nonlinear term is $v(x)=k_{2} x_{1} x_{2}\left[\begin{array}{ll}1 & -1\end{array}\right]^{\prime}$. Thus, for any $t \geq 0$ such that $y(t) \in \Omega$, the bound in Corollary 3 yields

$$
\begin{aligned}
|x(t, \varepsilon)-y(t, \varepsilon)|_{1, D} & \leq \int_{0}^{t} e^{-\eta(t-s)}|v(\exp (A s) \varepsilon)|_{1, D} \mathrm{~d} s \\
& =\int_{0}^{t} e^{-\eta(t-s)}|D v(\exp (A s) \varepsilon)|_{1} \mathrm{~d} s \\
& =k_{2}(d+1) \int_{0}^{t} e^{-\eta(t-s)} y_{1}(s) y_{2}(s) \mathrm{d} s
\end{aligned}
$$


It is possible of course to plug in the expression for the solution of the $y$ system to obtain an explicit bound.

\section{Discussion}

We considered the problem of approximating the trajectories of a contractive system using a "simpler" system. Our main result is an integral bound on the approximation error that depends on the trajectories of the simpler system. We demonstrated several applications of this result using models from systems biology. These models were approximated using LTI systems accompanied with an explicit bound on the approximation error.

An important property of contractive systems is that they entrain to a periodic excitation, that is, if the excitation has period $T$ then all their trajectories converge to a unique periodic trajectory with period $T$. However, the proof of this property (see, e.g. [3]) provides no explicit information on this periodic trajectory. An interesting direction for further research may be to approximate this periodic trajectory using the periodic trajectory of a simpler system (e.g., a stable linear system with a periodic excitation). Another direction for further research is applying the ideas described above to compute guaranteed overapproximations of reachable sets for nonlinear continuoustime systems. These are important in many applications, e.g., safety verification (see [7] and the references therein).

\section{REFERENCES}

[1] W. Lohmiller and J.-J. E. Slotine, “On contraction analysis for non-linear systems,” Automatica, vol. 34, pp. 683-696, 1998.

[2] Z. Aminzare and E. D. Sontag, "Contraction methods for nonlinear systems: A brief introduction and some open problems," in Proc. 53rd IEEE Conf. on Decision and Control, Los Angeles, CA, 2014, pp. 3835-3847.

[3] G. Russo, M. di Bernardo, and E. D. Sontag, "Global entrainment of transcriptional systems to periodic inputs," PLOS Computational Biology, vol. 6, p. e1000739, 2010.

[4] G. Russo, M. di Bernardo, and E. D. Sontag, "A contraction approach to the hierarchical analysis and design of networked systems," IEEE Trans. Automat. Control, vol. 58, pp. 1328-1331, 2013.

[5] M. Arcak, "Certifying spatially uniform behavior in reaction-diffusion PDE and compartmental ODE systems," Automatica, vol. 47, no. 6, pp. 1219-1229, 2011.

[6] C. Desoer and H. Haneda, "The measure of a matrix as a tool to analyze computer algorithms for circuit analysis," IEEE Trans. Circuit Theory, vol. 19, pp. 480-486, 1972.

[7] J. Maidens and M. Arcak, "Reachability analysis of nonlinear systems using matrix measures," IEEE Trans. Automat. Control, vol. 60, no. 1, pp. 265-270, 2015. 
[8] F. Forni and R. Sepulchre, "A differential Lyapunov framework for contraction analysis," IEEE Trans. Automat. Control, vol. 59, no. 3, pp. 614-628, 2014.

[9] I. W. Sandberg, "On the mathematical foundations of compartmental analysis in biology, medicine, and ecology," IEEE Trans. Circuits and Systems, vol. 25, no. 5, pp. 273-279, 1978.

[10] L. Grüne, "Input-to-state dynamical stability and its Lyapunov function characterization," IEEE Trans. Automat. Control, vol. 47, pp. 1499-1504, 2002.

[11] — Asymptotic Behavior of Dynamical and Control Systems under Perturbation and Discretization, ser. Lecture Notes in Mathematics, Vol. 1783. Heidelberg: Springer, 2002.

[12] E. D. Sontag, "Contractive systems with inputs," in Perspectives in Mathematical System Theory, Control, and Signal Processing, J. Willems, S. Hara, Y. Ohta, and H. Fujioka, Eds. Berlin Heidelberg: Springer-Verlag, 2010, pp. $217-228$.

[13] H. L. Smith, Monotone Dynamical Systems: An Introduction to the Theory of Competitive and Cooperative Systems, ser. Mathematical Surveys and Monographs. Providence, RI: Amer. Math. Soc., 1995, vol. 41.

[14] S. Reuveni, I. Meilijson, M. Kupiec, E. Ruppin, and T. Tuller, "Genome-scale analysis of translation elongation with a ribosome flow model," PLOS Computational Biology, vol. 7, p. e1002127, 2011.

[15] Y. Zarai, M. Margaliot, and T. Tuller, "Explicit expression for the steady-state translation rate in the infinite-dimensional homogeneous ribosome flow model," IEEE/ACM Trans. Computational Biology and Bioinformatics, vol. 10, pp. 1322-1328, 2013.

[16] Margaliot, M. and Tuller, T., "Ribosome flow model with positive feedback," J. Royal Society Interface, vol. 10, p. 20130267, 2013.

[17] M. Margaliot and T. Tuller, "Stability analysis of the ribosome flow model," IEEE/ACM Trans. Computational Biology and Bioinformatics, vol. 9, pp. 1545-1552, 2012.

[18] M. Margaliot, E. D. Sontag, and T. Tuller, "Entrainment to periodic initiation and transition rates in a computational model for gene translation," PLoS ONE, vol. 9, no. 5, p. e96039, 2014.

[19] A. Raveh, Y. Zarai, M. Margaliot, and T. Tuller, "Ribosome flow model on a ring," IEEE/ACM Trans. Computational Biology and Bioinformatics, vol. 12, no. 6, pp. 1429-1439, 2015.

[20] A. Raveh, M. Margaliot, E. D. Sontag, and T. Tuller, "A model for competition for ribosomes in the cell," J. Royal Society Interface, vol. 13, no. 116, 2016.

[21] G. Poker, M. Margaliot, and T. Tuller, "Sensitivity of mRNA translation,” Sci. Rep., vol. 5, p. 12795, 2015.

[22] B. Alberts, A. Johnson, J. Lewis, M. Raff, K. Roberts, and P. Walter, Molecular Biology of the Cell, 5th ed. New York: Garland Science, 2007.

[23] J. Smillie, "Competitive and cooperative tridiagonal systems of differential equations," SIAM J. Mathematical Analysis, vol. 15 , pp. 530-534, 1984.

[24] M. Margaliot, E. D. Sontag, and T. Tuller, "Contraction after small transients," Automatica, vol. 67, pp. 178-184, 2016.

[25] D. Del Vecchio, A. J. Ninfa, and E. D. Sontag, "Modular cell biology: Retroactivity and insulation," Molecular Systems Biology, vol. 4, no. 1, p. 161, 2008. 